\title{
AUV Trajectory Optimization for an Optical Underwater Sensor Network in the Presence of Ocean Currents
}

\author{
Khadijeh Ali Mahmoodi \\ Department of Electrical and Electronics Engineering \\ Ozyeǧin University \\ Istanbul, Turkey, 34794 \\ Khadijeh.ali@ozu.edu.tr
}

\author{
Murat Uysal \\ Department of Electrical and Electronics Engineering \\ Ozyeğin University \\ Istanbul, Turkey, 34794 \\ Murat.uysal@ozyegin.edu.tr
}

\begin{abstract}
Autonomous underwater vehicles (AUVs) are instrumental for data offloading in underwater sensor networks (USNs). With high data rate capacity at transmission ranges in the order of several tens of meters, visible light communication (VLC) is well-positioned to serve as a wireless link between the AUV and sensor nodes. In this paper, we consider a USN network where an AUV is used for data retrieval from the sensors through VLC link. We formulate the design of optimal AUV trajectory as an optimization problem to minimize the AUV energy consumption under data rate constraints imposed by the VLC link and in the presence of ocean currents. Our numerical results demonstrate that our proposed trajectory is reactive to ocean currents and brings significant reductions in energy consumption and mission time of the AUVs, in particular for USN scenarios with a large number of sensor nodes.
\end{abstract}

Index Terms-Visible light communication, AUV trajectory, underwater sensor network.

\section{INTRODUCTION}

Underwater sensor networks (USNs) have been increasingly deployed in various maritime applications including pollution monitoring, tsunami warnings, underwater oil field detection, and valuable minerals explorations among others [1]. Autonomous underwater vehicles (AUVs) are particularly instrumental in USNs to retrieve data from sensor nodes [2]. In AUV-assisted USNs, the AUV travels around to gather data from sensor nodes, stores it and transfers the information to the surface buoy. For data transfer between the AUV and sensors, the common choice is acoustic signaling [3] and acoustic modems are already available from a number of vendors [4]. While acoustic communication enables transmission over long ranges (in the order of several kilometers), it suffers from several disadvantages such as low data rates (in the order of tens of $\mathrm{Kb} / \mathrm{s}$ ) and low propagation speed $(1500 \mathrm{~m} / \mathrm{s})$ [5]. To address such

This work has been funded by the European Union's Horizon 2020 research and innovation programme under the Marie Skłodowska Curie grant agreement ENLIGHTEM No. 814215. challenges associated with acoustic transmission, visible light communication (VLC) has emerged as an alternative underwater wireless connectivity solution. VLC offers high data rates in the order of $\mathrm{Gb} / \mathrm{s}$, albeit at short and medium distances in the order of several tens of meters [6]. Such a transmission range and high data rate capability makes VLC a strong candidate for AUV-to-sensor communication.

In AUV operations, the major limiting constraint is power consumption since they rely on batteries with limited lifetimes. Therefore, the choice of efficient trajectories directly impacts the mission planning of AUVs. Predefined trajectories, as the name implies, are planned before the AUV starts its mission. In [2], [7]-[10], elliptical, circular, and lawnmower patterns were investigated as predefined AUV trajectories in acoustic USNs. Since the AUV must complete the defined trajectory, whether or not there are sensors around, the energy efficiency of predefined trajectories remains low in particular for large mission areas with randomly distributed sensor nodes.

Another choice is reactive trajectories where the AUV path is corrected and refined in real-time to cope with the sudden changes during the operation. In the literature, there have been some works on reactive trajectory optimization assuming both acoustic [11]-[14] and VLC [15] signaling. In [11], [12], trajectory planning of the AUV is formulated as a traveling salesman problem (TSP) to minimize the AUV travel time, and the ant colony algorithm is applied to solve the TSP. In [13], [14] TSP algorithm is proposed to define the path of the AUV with the objective of maximizing the value of information (VoI) from the sensors. In [15], the trajectory finding problem of the AUV is solved using a greedy algorithm under VoI constraints.

The abovementioned papers on reactive trajectories [11][15] simply assume that the AUV follows a straight path between each two sensor nodes without any deviations. However, in real-life conditions, due to ocean currents, that might not be possible. Trajectory optimization of standalone AUVs in the presence of ocean currents was addressed in [16]-[18]. However, these papers focus on stand-alone 
AUVs which move from a pre-defined start point to an endpoint. They do not take into account either the distribution of sensors in USN or signaling aspects between the AUV and the sensor.

In this paper, we consider a VLC-based USN network where the AUV is used for data retrieval from the sensors. Our objective is to determine the optimal trajectory of the AUV in the presence of ocean currents under the constraints of communication requirements. For trajectory definition, we formulate an optimization problem to minimize the energy consumption of the AUV. The communication constraint imposes a minimum required data rate for the VLC link between the AUV and each sensor node. This, therefore, dictates that the AUV needs to be at a certain distance from the sensor node. The optimal solution for the general case (i.e., where the order of sensor node visits is not defined) is very difficult, if not impossible. Therefore, we resort to a sub-optimal solution where we first determine the optimum sequence order of the sensor nodes to be visited, then optimize the trajectory between each pair of adjacent nodes for the given order of node visits. Our numerical results demonstrate that the proposed trajectory brings energy savings and decreases mission time in the presence of ocean currents.

The rest of the paper is organized as follows: In Section II, we present the system model. In Section III, we formulate the trajectory optimization problem to minimize energy consumption. In Section IV, we provide numerical results and finally conclude in Section V.

\section{System Model}

As illustrated in Fig.1, we consider a USN with $F$ sensors located at a depth of $Z_{\mathrm{s}}$ in an area of $G \mathrm{Km} \times H \mathrm{Km}$. The location of the sensor $\mathrm{S}_{i}, i=1,2, \ldots, F$ is defined by the position vector $\mathbf{p}_{S_{i}}=\left(x_{S_{i}}, y_{S_{i}}, z_{S_{i}}\right)$. AUV visits each sensor node once to retrieve data and returns to the initial point to transmit the aggregated data to a central node.

Let $T_{i}$ denote the travel time of the AUV from the sensor $\mathrm{S}_{i}$ to the sensor $\mathrm{S}_{i+1}$. This is divided into $N$ equallength time slots with a duration of $\Delta t_{i}=T_{i} / N$. If $\Delta t_{i}$ is chosen sufficiently small, the location of the AUV can be assumed to be fixed during each time slot. Thus, the position of the AUV at the $n^{\text {th }}$ slot, $n=1,2, \ldots, N$, during its travel from $\mathrm{S}_{i}$ to $\mathrm{S}_{i+1}$ can be described by the vector $\mathbf{p}_{\mathrm{A}_{i}}[n]=\left(x_{\mathrm{A}_{i}}[n], y_{\mathrm{A}_{i}}[n], z_{\mathrm{A}_{i}}[n]\right)$. Under the assumption that the AUV moves at a fixed depth, we can set $z_{\mathrm{A}_{i}}[n]=Z_{\mathrm{A}}<Z_{\mathrm{s}}$. Furthermore, we assume that the AUV moves with a constant speed at the horizontal plane [6]. Therefore, the velocity of the AUV is given by $\nu_{\mathrm{A}}[n]=\left(v_{\mathrm{A}_{x}}[n], v_{\mathrm{A}_{y}}[n], v_{\mathrm{A}_{z}}[n]\right)$. Accordingly, we formulate the discrete AUV state as

$$
\mathbf{p}_{\mathrm{A}_{i}}[n]=\mathbf{p}_{\mathrm{A}_{i}}[n-1]+\left(\nu_{\mathrm{o}}[n]+\nu_{\mathrm{A}}[n]\right) \Delta t_{i}[n], \quad \forall n
$$

where $\nu_{\mathrm{o}}[n]=\left[\nu_{\mathrm{o}_{x}}[n], \nu_{\mathrm{o}_{y}}[n]\right]$ is the random vector quantifying the ocean current speed in 2-dimensional space. Specifically, $\nu_{\mathrm{o}_{x}}[n]$ and $\nu_{\mathrm{o}_{y}}[n]$ are modeled as independent

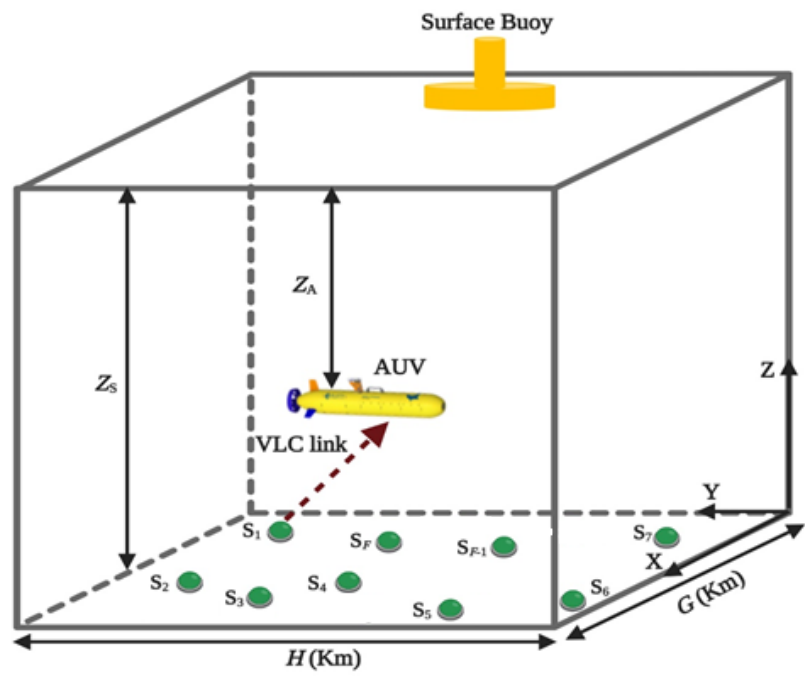

Fig. 1. AUV-assisted USN

Gaussian random variables with the mean value of $\alpha \mathrm{m} / \mathrm{s}$ and variance of $\beta$ [18].

Each sensor is equipped with a VLC transmitter while the AUV is equipped with a photodetector as the VLC receiver. We assume an intensity modulation-direct detection (IM/DD) system. The transmitted optical signal goes through the underwater propagation medium and reaches the destination. Based on the Beer-Lambert formula [19], the path loss term between the AUV and $S_{i}$ is given by

$$
h_{i}[n]=\exp \left(-c d_{i}[n]\right)
$$

where $c$ is the extinction coefficient and $d_{i}[n]=$ $\left\|\mathbf{p}_{\mathrm{A}_{i}}[n]-\mathbf{p}_{\mathrm{S}_{i}}\right\|$ is the link distance between the AUV and $\mathrm{S}_{i}$ in the $n^{\text {th }}$ time slot.

A closed-form expression for information rate in IM/DD systems is not available in the literature [20]. A lower bound of the information rate (in bits/s/Hz) is expressed as [21]

$$
R_{i}[n] \geq \frac{1}{2} \log _{2}\left(1+\frac{e}{2 \pi \sigma^{2}}\left(r h_{i}[n] P_{t}\right)^{2}\right)
$$

where $r$ is the detector's responsivity, $P_{\mathrm{t}}$ is the transmit power, and $\sigma^{2}=N_{0} B$ is the noise variance. Here, $B$ is bandwidth of the receiver, and $N_{0}$ is the noise power spectral density.

\section{PRoblem Formulation}

In this section, we aim to determine the optimal trajectory of the AUV in the sense of minimizing its energy consumption. The power consumption of an AUV in each time slot can be calculated by the summation of propulsion power $\Phi_{\text {prop }}$ and hotel load $\Phi_{\mathrm{H}}$ as [22]

$$
\Phi_{\mathrm{A}}[n]=\Phi_{\text {prop }}[n]+\Phi_{\mathrm{H}}
$$

Hotel load is the power consumed by all subsystems other than propulsion and is typically negligible in comparison with $\Phi_{\text {prop }}$ [23]. Propulsion power can be calculated as [24]

$$
\Phi_{\text {prop }}[n]=\frac{\rho}{2 \eta_{\mathrm{p}}} C_{\mathrm{D}} A_{\mathrm{s}}\left\|\nu_{\mathrm{A}}[n]\right\|^{3}
$$


where $\|$.$\| denotes the Euclidean vector norm and \rho$ is the density of water, $\eta_{\mathrm{p}}$ is the efficiency of the propulsion system, and $C_{\mathrm{D}}$ is the drag coefficient of the AUV. $A_{\mathrm{s}}$ is the wetted surface area of the AUV and is given by [22]

$$
\begin{aligned}
& A_{\mathrm{s}}=2 \pi \frac{D_{\mathrm{s}}{ }^{2}}{4}\left(1+\frac{L}{D_{\mathrm{s}} \sqrt{1-D_{\mathrm{s}}{ }^{2} / L^{2}}} \sin ^{-1}\left(\sqrt{1-D_{\mathrm{s}}{ }^{2} / L^{2}}\right)\right) \\
& +A_{\text {wings }}
\end{aligned}
$$

where $D_{\mathrm{s}}=\sqrt{6 m / \rho \pi L}$ is the diameter of the AUV, $m$ is the mass, $L$ is the length of the AUV, and $A_{\text {wings }}$ is the surface area of the AUV's wings.

The total energy consumption of the AUV to complete the mission (i.e., visiting $F$ sensors in the mission area) can be calculated as

$$
E_{\mathrm{tot}}=\sum_{i=1}^{F} \sum_{n=1}^{N} \Phi_{\mathrm{A}}[n] \Delta t_{i}[n]
$$

Replacing (1) and (4) in (7), we obtain

$$
E_{\text {tot }}=\left(\frac{\rho}{2 \eta_{\mathrm{p}}} C_{\mathrm{D}} A_{\mathrm{s}}\left\|\nu_{\mathrm{A}}\right\|^{3}+\Phi_{\mathrm{H}}\right) \sum_{i=1}^{F} \sum_{n=1}^{N} \frac{\left\|\mathbf{p}_{\mathrm{A}_{i}}[n]-\mathbf{p}_{\mathrm{A}_{i}}[n-1]\right\|}{\left\|\nu_{\mathrm{o}}+\nu_{\mathrm{A}}\right\|}
$$

Since the AUV's speed is assumed to be constant, the energy-optimal trajectory is equal to time-optimal trajectory [18]. In other words, our optimization problem reduces to the minimization of mission time. Let $T=\sum_{i=1}^{F} \sum_{n=1}^{N} \Delta t_{i}[n]$ denote the mission time which defines the total travel time starting from the first sensor node $S_{1}$ and returning to it after completing the mission. Let $\mathbf{P}_{\mathrm{A}}=\left[\mathbf{p}_{\mathrm{A}_{1}}[1] \mathbf{p}_{\mathrm{A}_{1}}[2] \ldots\right.$ $\mathbf{p}_{\mathrm{A}_{1}}[N] \mathbf{p}_{\mathrm{A}_{2}}[1] \mathbf{p}_{\mathrm{A}_{2}}[2] \ldots \mathbf{p}_{\mathrm{A}_{2}}[N] \ldots . . \mathbf{p}_{\mathrm{A}_{F}}[1] \mathbf{p}_{\mathrm{A}_{F}}[2] \ldots$

$\left.\mathbf{p}_{\mathrm{A}_{F}}[N] \mathbf{p}_{\mathrm{A}_{1}}[1]\right]$ denote the AUV trajectory. Accordingly, we can express the optimization problem as

$$
\begin{aligned}
& \min _{\mathbf{p}_{\mathrm{A}}} T \\
& \text { s.t. } R_{i}[n] \geq R_{\mathrm{th}}
\end{aligned}
$$

The constraint imposes a minimum required data rate (denoted by $R_{\mathrm{th}}$ ) for transfer between the AUV and each sensor node. Therefore, the AUV needs to be at a certain distance $d_{\mathrm{th}}$ from the sensor nodes to satisfy this condition, i.e., $\left\|\mathbf{p}_{\mathrm{A}_{i}}[n]-\mathbf{p}_{\mathrm{S}_{i}}\right\| \leq d_{\mathrm{th}}$. Based on (2) and (3), we can obtain $d_{\mathrm{th}}$ as

$$
d_{\mathrm{th}} \leq \frac{1}{2 c} \ln \left[\left(\frac{2 \pi \sigma^{2}}{e r^{2} P_{\mathrm{t}}}\left(2^{R_{\mathrm{th}}}-1\right)\right)^{-1}\right]
$$

Unfortunately, the optimal solution of (9) for the general case (i.e., where the order of sensor node visits is not defined) is very difficult, if not impossible. Therefore, we resort to a sub-optimal solution. First, we determine the optimum sequence order of the sensor nodes to be visited, which can be considered as a large-scale trajectory optimization. Once the order of sensor node visits is determined, we deal with the small-scale optimization problem that optimizes the path between each pair of adjacent nodes to minimize the overall mission time. It should be noted
TABLE I

SYSTEM AND CHANNEL PARAMETERS

\begin{tabular}{|l|l|l|}
\hline Parameter & Variable & Value \\
\hline Mission area & $G \times H$ & $20 \times 20 \mathrm{Km}^{2}[21]$ \\
\hline Number of sensor nodes & $F$ & 20 \\
\hline Depth of the sensors & $Z_{\mathrm{s}}$ & $250 \mathrm{~m}$ \\
\hline Number of time slots & $N$ & 100 \\
\hline Mean of ocean current speed & $\alpha$ & $0.6 \mathrm{~m} / \mathrm{s} \mathrm{[19]}$ \\
\hline Variance of ocean current speed & $\beta$ & $0.01[18]$ \\
\hline Transmit power & $P_{\mathrm{t}}$ & $0.01 \mathrm{~W}[28]$ \\
\hline Detector responsivity & $r$ & $0.5[28]$ \\
\hline Noise power spectral density & $N_{0}$ & $10^{-19} \mathrm{~W} / \mathrm{Hz}[28]$ \\
\hline Bandwidth of the receiver & $B$ & $100 \mathrm{MHz}$ \\
\hline Extinction coefficient for clear ocean & $c$ & $0.15[28]$ \\
\hline
\end{tabular}

TABLE II

SPECIFICATIONS OF THE AUV [29]

\begin{tabular}{|l|l|l|}
\hline Parameter & Variable & Value \\
\hline Length of the AUV & $L$ & $1.8 \mathrm{~m}$ \\
\hline Mass of the AUV & $m$ & $50 \mathrm{Kg}$ \\
\hline Diameter of the AUV & $D_{\mathrm{s}}$ & $0.2 \mathrm{~m}$ \\
\hline Wetted surface area & $A_{\mathrm{s}}$ & $47.18 \mathrm{~m}^{2}$ \\
\hline Drag coefficient & $C_{\mathrm{D}}$ & 0.0064 \\
\hline Efficiency of the propulsion system & $\eta_{\mathrm{p}}$ & $100 \%$ \\
\hline Velocity of the AUV & $\| \nu_{\mathrm{A}}$ & $0.5 \mathrm{~m} / \mathrm{s}$ \\
\hline Depth of the AUV & $Z_{\mathrm{A}}$ & $220 \mathrm{~m}$ \\
\hline Water density & $\rho$ & $997 \mathrm{Kg} / \mathrm{m}^{3}$ \\
\hline
\end{tabular}

that if ocean currents are ignored, our solution reduces to large-scale optimization.

For large-scale optimization, we formulate the problem as a TSP to minimize the mission time assuming direct paths between each two nodes [25]. For the solution of TSP, we use the genetic algorithm (GA) toolbox in MATLAB [26] which yields the order of sensor node visits. Then, for the determined order of sensor nodes, we numerically solve (9) using fmincon function of the MATLAB optimization toolbox [27]. This function is based on the trust-region algorithm and is defined to carry out the optimization by using the present information and then to repeat the process over and over. Accordingly, with knowledge of the start and the endpoints of the movement and the underwater current speed in each time slot, the best trajectory between two sensor nodes is determined.

\section{NumericAl Results}

In this section, we present our numerical results for the optimal trajectory and discuss the energy and time savings made possible through the optimization. We consider an area of $G \times H=20 \mathrm{Km} \times 20 \mathrm{Km}$ where the sensors are located at the seabed with a depth of $Z_{\mathrm{s}}=250 \mathrm{~m}$ and the AUV swims at a fixed depth of $Z_{\mathrm{A}}=220 \mathrm{~m}$. Unless otherwise stated, system and channel parameters are provided in Table I. AUV specifications are further provided in Table II. For the solution of TSP algorithm, the number of populations and the number of iterations in the genetic algorithm are set as 100 and 10000, respectively.

In Fig.2, we consider a USN where $F=20$ sensor nodes are uniformly distributed on a rectangular grid pat- 


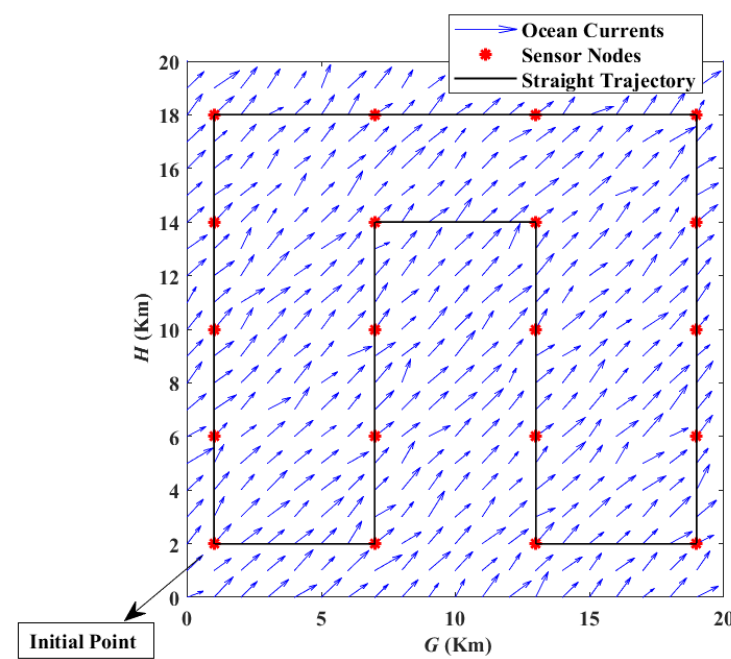

(a)

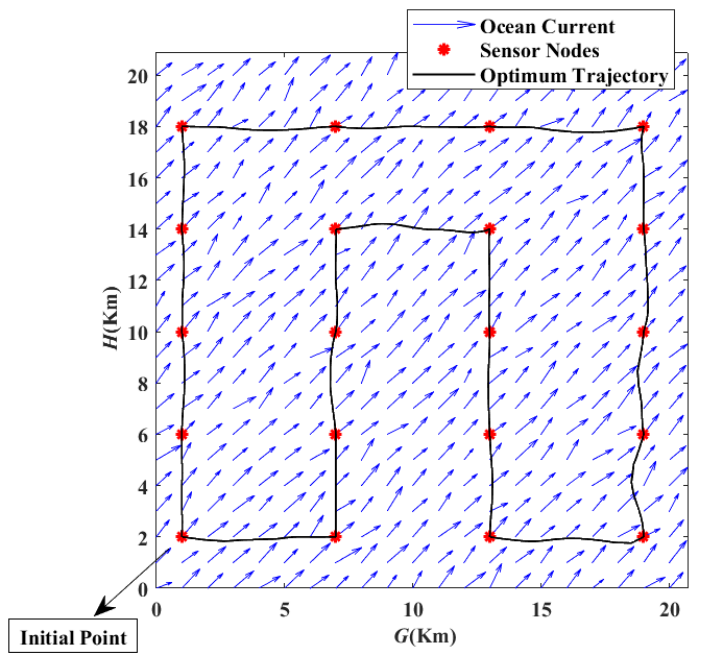

(b)

Fig. 2. AUV trajectories for uniformly distributed sensors: a) Straight trajectory (i.e., only large-scale optimization), b) Optimum trajectory

tern. As a benchmark, we include the case where AUV follows a straight trajectory between every two nodes as illustrated in Fig.2.a (i.e., this is simply based on the largescale optimization results). Under the assumption of AUV velocity of $\left\|\nu_{\mathrm{A}}\right\|=0.5 \mathrm{~m} / \mathrm{s}$, it takes $T=60$ hours to complete the mission, which means $E_{\text {tot }}=4.06 \mathrm{MJ}$ of energy consumption. Our proposed trajectory found from the solution of (9) is presented in Fig.2.b. It is observed that the AUV does not any longer follow a straight path because the AUV selects its path reactive to ocean currents. In particular, for most cases, the AUV avoids to swim towards the opposite direction of ocean currents whenever possible to save energy. Using the proposed trajectory, it takes $T=57.5$ hours and $E_{\text {tot }}=3.91 \mathrm{MJ}$ of energy consumption to complete the mission. Comparison with the benchmark in Fig.2.a demonstrates that the AUV saves 2.5 hours to complete its mission, which means $169 \mathrm{KJ}$ of saving energy. This obviously indicates the superiority of the proposed trajectory.

In Fig.3, we consider a USN where $F=20$ sensors are randomly distributed. The results in the following are obtained based on averaging over 100 trials while the figure shows only one trial as an example. In each trial, we change the location of randomly distributed sensor nodes while the ocean currents distribution remains the same. For the AUV velocity of $\left\|\nu_{\mathrm{A}}\right\|=0.5 \mathrm{~m} / \mathrm{s}$, it takes $T=57.5$ hours and $E_{\mathrm{tot}}=3.89 \mathrm{MJ}$ of energy consumption to complete the mission for straight trajectory (i.e., only large-scale optimization which effectively ignores the ocean currents). This reduces to $T=54.9$ hours and $E_{\text {tot }}=3.72 \mathrm{MJ}$ for the proposed trajectory. This indicates an average of 2.6 hours reduction in the mission time and an average energy saving of $180 \mathrm{KJ}$.

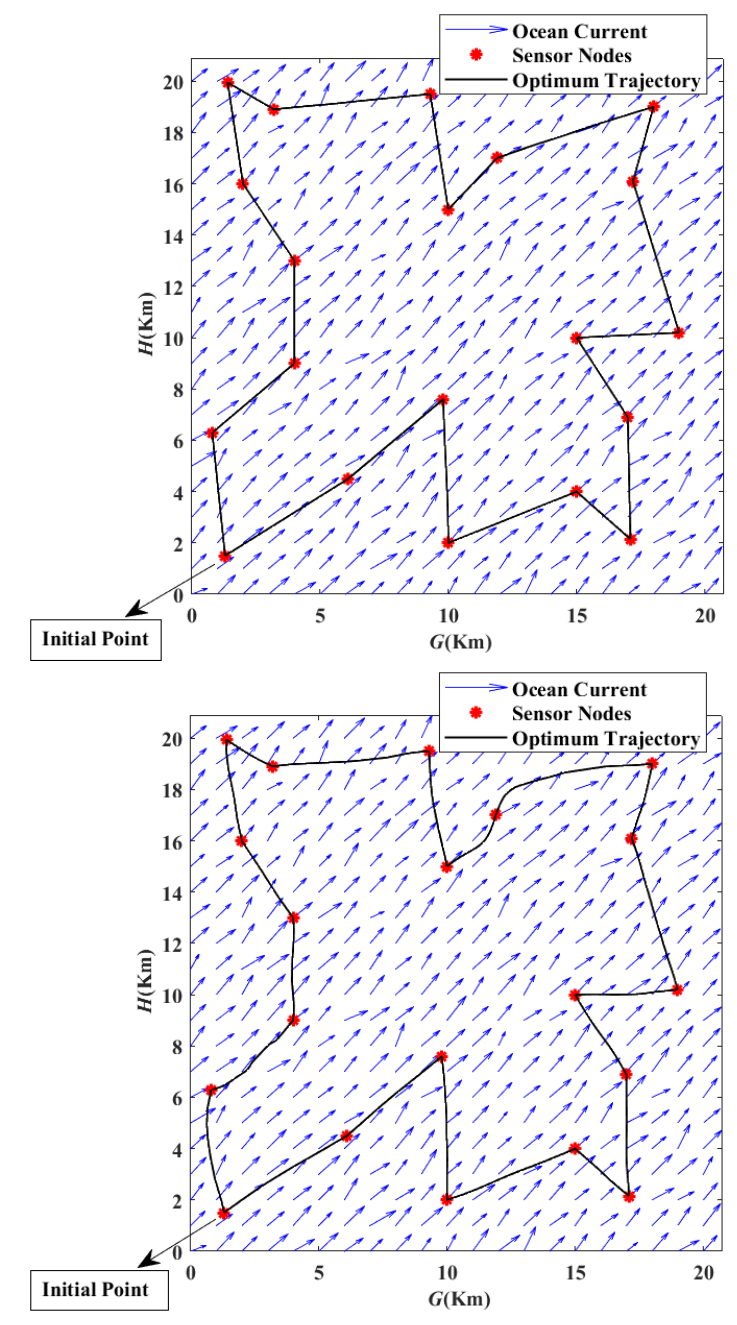

(a)

Fig. 3. AUV trajectories for randomly distributed sensors: a) Straight trajectory (i.e., only large-scale optimization), b) Optimum trajectory

In Fig.4, we investigate the number of sensor nodes on the energy consumption and mission time. Results are averaged of 100 trials and the same conditions in Fig.3 are considered. The number of sensor nodes ranges from $F=5$ to $F=50$. For $F=5$ sensor nodes, the mission time and the corresponding energy consumption for the straight trajectory are respectively $T=27.6$ hours and 


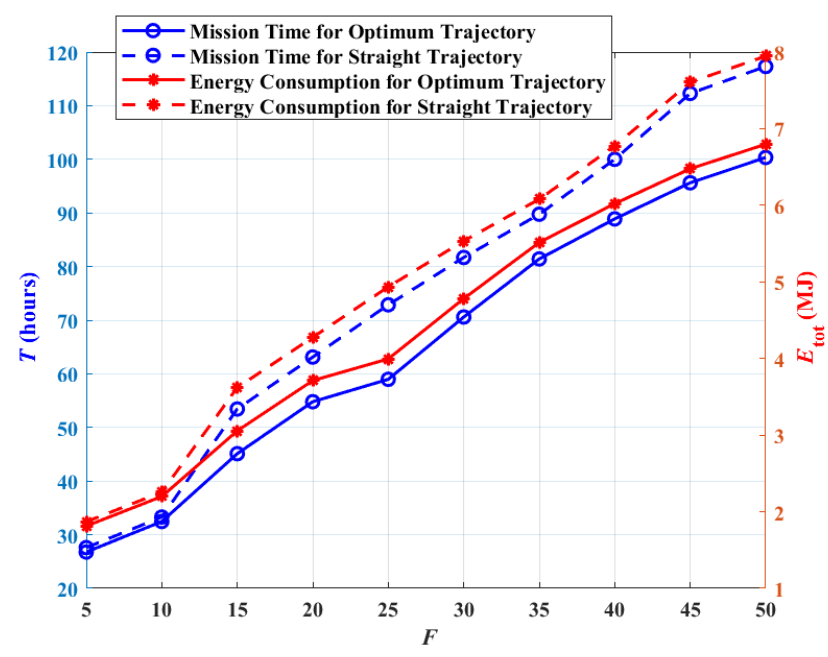

Fig. 4. Effect of the number of sensor nodes on the mission time and energy consumption

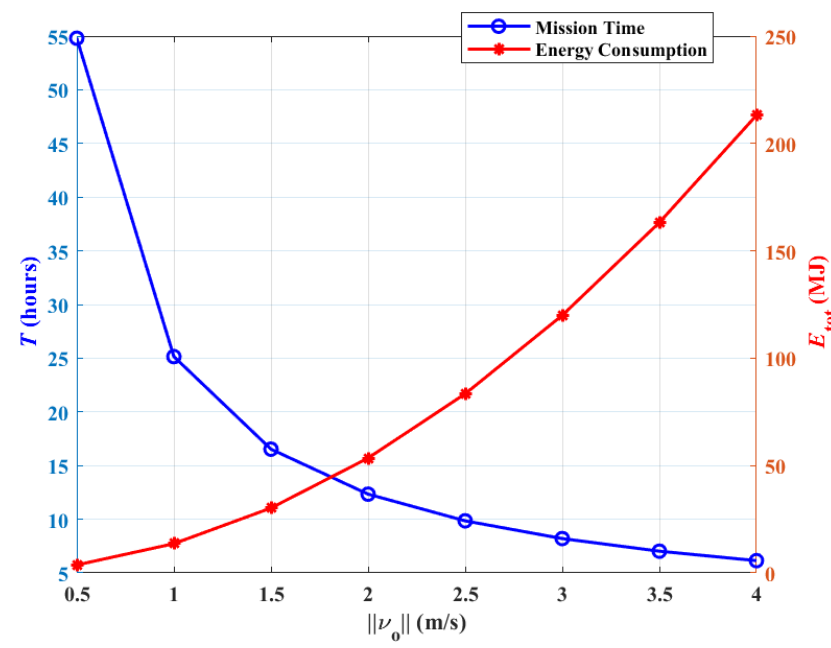

Fig. 5. Effect of AUV velocity on the mission time and energy consumption (Only optimal trajectory is considered.)

$E_{\text {tot }}=1.86$ MJ. These reduce to $T=26.7$ hours and $E_{\text {tot }}=1.81 \mathrm{MJ}$ for the proposed trajectory. This indicates an improvement of $3.3 \%$ in travel time and $2.7 \%$ in energy consumption. For $F=50$ sensor nodes, it requires $T=117.3$ hours and $T=100.1$ hours respectively for straight and optimum trajectories, indicating an improvement of $14.7 \%$ in travel time. We have $E_{\text {tot }}=7.94 \mathrm{MJ}$ and $E_{\text {tot }}=6.7 \mathrm{MJ}$ for straight and proposed trajectories which yields an improvement of $15.6 \%$ in energy consumption. It can be readily checked that the improvement increases as the number of sensor nodes increases.

In Fig.5, we investigate the effect of AUV's velocity on the energy consumption and mission time. Other assumptions are the same as in those in Fig.3. It is observed that as the velocity increases, the mission time decreases while the energy consumption increases. For example, the mission time for $\left\|\nu_{\mathrm{A}}\right\|=4 \mathrm{~m} / \mathrm{s}$ is $T=6.2$ hours which is 48.3 hours less than that in $\left\|\nu_{\mathrm{A}}\right\|=0.5 \mathrm{~m} / \mathrm{s}$ (considered in Fig.3). Accordingly, the energy consumption reaches to $E_{\text {tot }}=213 \mathrm{MJ}$ from $E_{\text {tot }}=3.72 \mathrm{MJ}$. This also indicates the need to determine a trade-off between the energy and mission time requirements. For the given scenario under consideration, it can be readily checked from Fig. 5 that $\left\|\nu_{\mathrm{A}}\right\|=1.8 \mathrm{~m} / \mathrm{s}$ gives such a good trade-off where the plots of mission time and energy consumption intersect.

\section{CONCLUSION}

In this paper, we considered a USN network where the AUV visits sensor nodes for data retrieval through a VLC link. We formulated the design of optimal AUV trajectory as an optimization problem to minimize the AUV energy consumption (equivalently, to minimize mission time for constant speed). The optimization problem was numerically solved under data rate constraints imposed by the VLC link and in the presence of ocean currents. Our results demonstrated that the proposed trajectory is reactive to ocean currents and brings energy savings and decreases mission time. These improvements are more pronounced for larger number of sensor nodes. We further investigated the effect of AUV speed on the trajectory and demonstrated that proper choice of the speed is important to find the best trade-off between mission time and energy consumption.

\section{REFERENCES}

[1] K. K. Gola and B. Gupta, "Underwater sensor networks: 'comparative analysis on applications, deployment and routing techniques,," IET Communications, vol. 14, no. 17, pp. 2859-2870, 2020.

[2] H. Nam, "Data-gathering protocol-based auv path-planning for longduration cooperation in underwater acoustic sensor networks," IEEE sensors journal, vol. 18, no. 21, pp. 8902-8912, 2018.

[3] E. M. Sozer, M. Stojanovic, and J. G. Proakis, "Underwater acoustic networks," IEEE journal of oceanic engineering, vol. 25, no. 1, pp. 72-83, 2000.

[4] S. Sendra, J. Lloret, J. M. Jimenez, and L. Parra, "Underwater acoustic modems," IEEE Sensors Journal, vol. 16, no. 11, pp. 40634071, 2015.

[5] R. Su, D. Zhang, C. Li, Z. Gong, R. Venkatesan, and F. Jiang, "Localization and data collection in auv-aided underwater sensor networks: Challenges and opportunities," IEEE Network, vol. 33, no. 6, pp. 86-93, 2019.

[6] F. Akhoundi, M. V. Jamali, N. B. Hassan, H. Beyranvand, A. Minoofar, and J. A. Salehi, "Cellular underwater wireless optical cdma network: Potentials and challenges," IEEE Access, vol. 4, pp. 4254 4268, 2016

[7] N. Javaid, N. Ilyas, A. Ahmad, N. Alrajeh, U. Qasim, Z. A. Khan, T. Liaqat, and M. I. Khan, "An efficient data-gathering routing protocol for underwater wireless sensor networks," Sensors, vol. 15, no. 11, pp. 29 149-29 181, 2015.

[8] A. Ahmad, A. Wahid, and D. Kim, "Aeerp: Auv aided energy efficient routing protocol for underwater acoustic sensor network," in Proceedings of the 8th ACM workshop on Performance monitoring and measurement of heterogeneous wireless and wired networks, 2013, pp. 53-60.

[9] N. Wang and J. Wu, "Trajectory scheduling for timely data report in underwater wireless sensor networks," in 2015 IEEE Global Communications Conference (GLOBECOM). IEEE, 2015, pp. 1-6.

[10] N. Ilyas, N. Javaid, Z. Iqbal, M. Imran, Z. A. Khan, U. Qasim, and M. Shoaib, "Aaeerp: Advanced auv-aided energy efficient routing protocol for underwater wsns," in 2015 IEEE 29th International Conference on Advanced Information Networking and Applications. IEEE, 2015, pp. 77-83. 
[11] X. Zhuo, M. Liu, Y. Wei, G. Yu, F. Qu, and R. Sun, "Auvaided energy-efficient data collection in underwater acoustic sensor networks," IEEE Internet of Things Journal, 2020.

[12] C. Qin, J. Du, J. Wang, and Y. Ren, "A hierarchical information acquisition system for auv assisted internet of underwater things," IEEE Access, vol. 8, pp. 176089-176 100, 2020.

[13] F. A. Khan, S. A. Khan, D. Turgut, and L. Bölöni, "Scheduling multiple mobile sinks in underwater sensor networks," in 2015 IEEE 40th Conference on Local Computer Networks (LCN). IEEE, 2015, pp. $149-156$.

[14] G. A. Hollinger, S. Choudhary, P. Qarabaqi, C. Murphy, U. Mitra, G. S. Sukhatme, M. Stojanovic, H. Singh, and F. Hover, "Underwater data collection using robotic sensor networks," IEEE Journal on Selected Areas in Communications, vol. 30, no. 5, pp. 899-911, 2012.

[15] P. Gjanci, C. Petrioli, S. Basagni, C. A. Phillips, L. Bölöni, and D. Turgut, "Path finding for maximum value of information in multimodal underwater wireless sensor networks," IEEE Transactions on Mobile Computing, vol. 17, no. 2, pp. 404-418, 2017.

[16] M. Soulignac, "Feasible and optimal path planning in strong current fields," IEEE Transactions on Robotics, vol. 27, no. 1, pp. 89-98, 2010.

[17] A. A. R. Al Makdah, N. Daher, D. Asmar, and E. Shammas, "Threedimensional trajectory tracking of a hybrid autonomous underwater vehicle in the presence of underwater current," Ocean Engineering, vol. 185, pp. 115-132, 2019.

[18] X. Yao, F. Wang, J. Wang, and X. Wang, "Bilevel optimizationbased time-optimal path planning for auvs," Sensors, vol. 18, no. 12, p. 4167, 2018.

[19] C. D. Mobley, B. Gentili, H. R. Gordon, Z. Jin, G. W. Kattawar, A. Morel, P. Reinersman, K. Stamnes, and R. H. Stavn, "Comparison of numerical models for computing underwater light fields," Applied Optics, vol. 32, no. 36, pp. 7484-7504, 1993.

[20] A. A. Farid and S. Hranilovic, "Upper and lower bounds on the capacity of wireless optical intensity channels," in 2007 IEEE International Symposium on Information Theory. IEEE, 2007, pp. 2416-2420.

[21] J.-B. Wang, Q.-S. Hu, J. Wang, M. Chen, and J.-Y. Wang, "Tight bounds on channel capacity for dimmable visible light communications," Journal of Lightwave Technology, vol. 31, no. 23, pp. 37713779, 2013.

[22] A. Phillips, M. Haroutunian, A. J. Murphy, S. Boyd, J. Blake, and G. Griffiths, "Understanding the power requirements of autonomous underwater systems, part i: An analytical model for optimum swimming speeds and cost of transport," Ocean Engineering, vol. 133, pp. 271-279, 2017.

[23] M. E. Furlong, S. D. McPhail, and P. Stevenson, "A concept design for an ultra-long-range survey class auv," in OCEANS 2007-Europe. IEEE, 2007, pp. 1-6.

[24] J. G. Bellingham and J. S. Willcox, "Optimizing auv oceanographic surveys," in Proceedings of Symposium on Autonomous Underwater Vehicle Technology. IEEE, 1996, pp. 391-398.

[25] X. Chen, Y. Liu, X. Li, Z. Wang, S. Wang, and C. Gao, "A new evolutionary multiobjective model for traveling salesman problem," IEEE Access, vol. 7, pp. 66964-66979, 2019.

[26] J. Kirk. (2020) Traveling Salesman Problem (TSP) Genetic Algorithm Toolbox kernel description. [Online]. Available: https://github.com/rubikscubeguy/matlab-tsp-ga/releases/tag/v3.1.0

[27] J.-S. Lee and K.-H. Yu, "Optimal path planning of solar-powered uav using gravitational potential energy," IEEE Transactions on Aerospace and Electronic Systems, vol. 53, no. 3, pp. 1442-1451, 2017.

[28] M. Elamassie, F. Miramirkhani, and M. Uysal, "Channel modeling and performance characterization of underwater visible light communications," in 2018 IEEE International Conference on Communications Workshops (ICC Workshops). IEEE, 2018, pp. 1-5.

[29] http://www.teledynemarine.com/gavia-auv?brandid=9. 